\title{
Reproducible and Reliable Modified Technique of Surgical Aortic Valve Replacement That Decreases the Incidence of Permanent Pace Maker Implantation
}

\author{
Nadine Kawkabani ${ }^{1}$, , Omar Boustros ${ }^{2}$, Rita Farah ${ }^{3}$, Roula Darwish ${ }^{1}$, Moussa Abi Ghanem ${ }^{2}$, \\ Hassan Souidan $^{2}$, Hiba Zeidh ${ }^{2}$, Mohammad Hadi El Charif ${ }^{2}$, Bassam Abou Khalil ${ }^{2}$ \\ ${ }^{1}$ Cardiothoracic Anesthesia Department, Saint George Hospital Medical Center, Balamand University, Saint George University, Beirut, \\ Lebanon \\ ${ }^{2}$ Cardiothoracic Surgery Department, Saint George Hospital, Balamand University, Saint George University, Beirut, Lebanon \\ ${ }^{3}$ Faculty of Pharmacy, Lebanese University, INSPECT, LB, Beirut, Lebanon
}

Email address:

nadine_kaoukabani@yahoo.com (N. Kawkabani)

${ }^{*}$ Corresponding author

\section{To cite this article:}

Nadine Kawkabani, Omar Boustros, Rita Farah, Roula Darwish, Moussa Abi Ghanem, Hassan Souidan, Hiba Zeidh, Mohammad Hadi El Charif, Bassam Abou Khalil. Reproducible and Reliable Modified Technique of Surgical Aortic Valve Replacement That Decreases the Incidence of Permanent Pace Maker Implantation. International Journal of Cardiovascular and Thoracic Surgery.

Vol. 6, No. 3, 2020, pp. 44-48. doi: 10.11648/j.ijcts.20200603.12

Received: March 1, 2020; Accepted: August 3, 2020; Published: August 20, 2020

\begin{abstract}
In the last few years, a significant increase in the number of patients with aortic stenosis requiring surgical or transcatheter aortic replacement (SAVR) or (TAVR) has been observed due to the larger aging population. Conduction disturbances requiring permanent pace maker implantation (PPMI) has been observed after SAVR and TAVR. In fact the incidence of PPMI following SAVR reached 11 to $13 \%$ while it occurred in 7 to $36 \%$ of patients undergoing TAVR. The majority of rhythm problems are secondary to a significant trauma to the conduction system. In order to decrease the incidence of PPMI in patients undergoing SAVR, we developed a modified technique of SAVR that we applied on a group of 63 patients (group B) and we compared the incidence of PPMI in this group to the one observed in a second group of 62 patients who underwent the classic SAVR (group A). It was significantly lower in group B (3.2\% vs $14.5 \%$ ). In conclusion, The low incidence of PPMI (3.2\%) observed in the modified SAVR group encourages us to recommend this technique in all patients undergoing biological SAVR especially that this technique is simple to apply highly reproducible and reliable. However, further multicenter and larger studies will help confirm our findings.
\end{abstract}

Keywords: Aortic Valve Replacement, New Technique, Incidence of Pace Maker Implantation

\section{Introduction}

A significant increase in the number of patients referred for surgical treatment for aortic valve disease has been observed in the last years due to improved diagnosis of valvular disease and population aging [1].

New onset complete atrio-ventricular block (AV block) needing permanent pace maker implantation (PPMI) is a widely recognized complication following aortic valve replacement (AVR) with an incidence ranging between 3 to $11.8 \%$ [1-3]. The mean time to PPMI post AVR ranges from
6 to 13 days [3].

The complete AV block might be due to a direct trauma and stress to the conduction system. In fact during aortic valve replacement, trauma might occur following debridement of a severely calcified aortic valve or placement of sutures especially in the triangular area that embeds the conduction system $[1,2,4]$.

To note that many factors has also been incriminated in increasing the incidence of complete $\mathrm{AV}$ block such as preoperative rhythm abnormalities, myocardial infarction, use of beta blockers and calcium channel blockers, perioperative electrolytes imbalance, surgery for aortic 
insufficiency and long bypass [1-4].

In order to decrease the incident of PPMI in patients undergoing surgical aortic valve replacement (SAVR), we developed a modified technique that allows the surgeon to minimize the stress on the AV conduction system then we conducted a study in order to analyze the impact of this new technique on the PPMI incidence post SAVR.

In fact, The study compares the PPMI in two groups of patients undergoing surgical biological AVR. In the first group the patients underwent the classic surgical AVR technique while in group $\mathrm{B}$, the patients underwent the modified technique. The effect of the surgical technique as well as other variables on the incidence of PPMI was analyzed.

\section{Methods}

\section{Population}

The perioperative course of 125 patients who underwent surgical biological aortic valve replacement in Saint George
University Medical Center between January 2016 and June 2019 was prospectively analyzed.

Data Collection

Data were collected using a questionnaire. Patients were evaluated- after obtaining an informed consent- before surgery and closely in the postoperative period till two months after surgery.

The Institutional review board (IRB) at Saint George university medical center approved the study.

Operative Technique

All surgical cases were performed by one surgeon. Biological aortic valve replacement was performed for the clinical indication of AVR such as aortic stenosis, calicifications... Participants were divided into two groups; Group A that included 62 patients who underwent the classic technique of AVR between January 2016 and June 2017 and group B that included 63 patients who underwent a modified technique of aortic valve replacement between July 2017 and June 2019. Demographic and characteristics data are shown in table 1 and table 2 .

Table 1. Demographics and characteristics data of the patients. Data is presented as $N(\%)$ or mean (standard deviation) as indicated; Level of significance is set at $p<0.05 ;$ AVR: Aortic valve replacemeny; CABG: Coronary artery bypass grafting; MVR: Mitral valve replacement; PAP: Pulmonary arterial pressure; LBBB: Left bundle branch block; RBBB: Right bundle branch block; AI: Aortic insufficiency.

\begin{tabular}{|c|c|c|c|c|}
\hline & PPMI (N=11) & No PPMI $(\mathrm{N}=114)$ & p value & Total \\
\hline Gender & & & 0.34 & \\
\hline Male & $3(27.3 \%)$ & $52(48.2 \%)$ & & 55 \\
\hline Female & $8(72.7 \%)$ & $62(51.8 \%)$ & & 70 \\
\hline Age, in years (Mean, SD) & $76 \pm 4$ & $72 \pm 7$ & 0.047 & \\
\hline Arterial Hypertension & & & 0.68 & \\
\hline Yes & $10(91 \%)$ & $91(80 \%)$ & & 101 \\
\hline No & $1(9 \%)$ & $23(20 \%)$ & & 24 \\
\hline Yes & 0 & $4(3.5 \%)$ & & 4 \\
\hline No & $11(100 \%)$ & $108(94.7 \%)$ & & 119 \\
\hline Pulmonary hypertension & & & 1 & \\
\hline Yes & $1(9 \%)$ & $17(15 \%)$ & & 18 \\
\hline No & $10(91 \%)$ & $97(85 \%)$ & & 107 \\
\hline Treatment with beta blocker & & & 0.73 & \\
\hline Yes & $7(64 \%)$ & $79(69 \%)$ & & 86 \\
\hline No & $4(36 \%)$ & $35(31 \%)$ & & 39 \\
\hline Yes & $6(54 \%)$ & $50(44 \%)$ & & 56 \\
\hline No & $5(46 \%)$ & $64(56 \%)$ & & 69 \\
\hline Treatment with CCB & & & 0.48 & \\
\hline Yes & $4(36 \%)$ & $29(25 \%)$ & & 33 \\
\hline No & $7(64 \%)$ & $85(75 \%)$ & & 92 \\
\hline NSR & & & 1 & \\
\hline Yes & $10(91 \%)$ & $100(88 \%)$ & & 110 \\
\hline No & $1(9 \%)$ & $14(12 \%)$ & & 15 \\
\hline History of atrial fibrillation & & & 1 & \\
\hline Yes & $1(9 \%)$ & $15(13 \%)$ & & 16 \\
\hline No & $10(91 \%)$ & $99(87 \%)$ & & 109 \\
\hline History of AV block & & & 1 & \\
\hline Yes & $11(100 \%)$ & $107(94 \%)$ & & 118 \\
\hline No & 0 & $7(6 \%)$ & & 7 \\
\hline LBBB & & & 1 & \\
\hline RBBB & & & 0.25 & \\
\hline Yes & $2(18 \%)$ & $9(8 \%)$ & & 11 \\
\hline No & $9(82 \%)$ & $105(92 \%)$ & & 114 \\
\hline AI & & & 1 & \\
\hline Yes & $4(9 \%)$ & $42(37 \%)$ & & 46 \\
\hline
\end{tabular}




\begin{tabular}{lllll}
\hline & PPMI (N=11) & No PPMI (N=114) & p value & Total \\
\hline $\begin{array}{l}\text { No } \\
\text { Annular dilation }\end{array}$ & $5(46 \%)$ & $55(48 \%)$ & & 60 \\
Yes & $1(9 \%)$ & $9(8 \%)$ & 1 & 10 \\
No & $10(91 \%)$ & $105(92 \%)$ & 0.34 & 115 \\
Annular calcification & 9 & 75 & \\
Yes & 2 & 39 & 0.17 & \\
No & & & 41 \\
Bicuspid valve & $3(27 \%)$ & $14(12 \%)$ & 17 \\
Yes & $8(73 \%)$ & $100(88 \%)$ & \\
No & & & 108 \\
\hline
\end{tabular}

Data is presented as $\mathrm{N}(\%)$ or mean (standard deviation) as indicated; Level of significance is set at $\mathrm{p}<0.05$; ACEI: angiotensin-converting-enzyme inhibitor CCB: calcium channel blocker; NSR: Normal sinus rhythm.

Table 2. Per- and post-operative characteristics.

\begin{tabular}{|c|c|c|c|c|}
\hline & PPMI (N=11) & No PPMI (N=114) & p value & Total \\
\hline Method & & & 0.025 & \\
\hline Classic & $9(81.8 \%)$ & $53(46.5 \%)$ & & 62 \\
\hline New & $2(18.2 \%)$ & $61(53.5 \%)$ & & 63 \\
\hline \multicolumn{5}{|c|}{ Cardiopulmonary bypass time (Mean, SD) } \\
\hline PAP & & & 0.8 & \\
\hline Yes & $6(54 \%)$ & $56(49 \%)$ & & 62 \\
\hline No & $5(46 \%)$ & $55(48 \%)$ & & 60 \\
\hline Potassium serum level & & & 1 & \\
\hline Normal & $11(100 \%)$ & $105(92 \%)$ & & 116 \\
\hline Low & 0 & $9(8 \%)$ & & 9 \\
\hline Calcium serum level & & & 0.69 & \\
\hline Normal & $10(91 \%)$ & $94(82 \%)$ & & 104 \\
\hline Low & $1(9 \%)$ & $20(18 \%)$ & & 21 \\
\hline Procedure & & & 0.9 & \\
\hline AVR & $7(64 \%)$ & $63(55 \%)$ & & 70 \\
\hline AVR \& CABG & $4(36 \%)$ & $41(36 \%)$ & & 45 \\
\hline AVR \& MVR & 0 & $10(9 \%)$ & & 10 \\
\hline
\end{tabular}

In the classic technique of AVR, the surgeon used pledgeted 2.0 ethibond with the pledgets applied below the annulus while in the modified technique the same 2.0 pledgeted ethibond sutures were put below the annulus except for 3-4 sutures at the commissure between the right and non coronary sinuses. They were applied above the annulus. (Figure 1 and Figure 2)

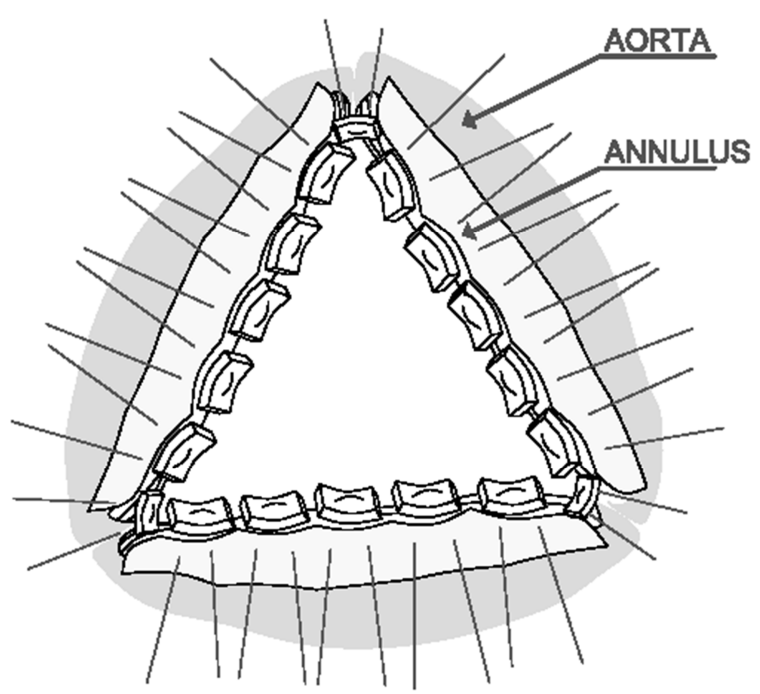

Figure 1. Classic technique of AVR.

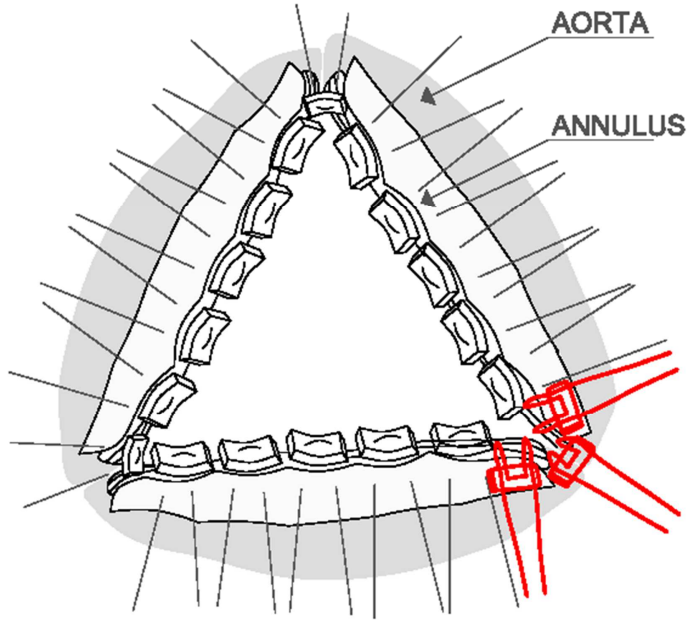

Figure 2. Modified surgical technique.

Postoperative follow up:

All patients were closely followed in the immediate post operative period till two months after surgery.

Post operative conduction disturbances in particular AV block and the subsequent need for a permanent pace maker were studied. Other complications such as hemodynamic instability, electrolytes imbalance. Were also analyzed.

Statistical analysis

We used the SPSS software version23.0 for data analysis. We used means with standard deviations and percentages to 
describe continuous and categorical variables. We used the Pearson chi-square $\left(\mathrm{X}^{2}\right)$ test to compare caterogical variables and when the expected values with cells were $<5$, the fisher exact test was used. We used the Student's t-test to compare means between two groups. A $p$ value $<0.05$ was considered statistically significant. We performed multivariate analysis to study the predictors of the need for pacemaker using a binary logistic regression model.

Results

Postoperatively, 9 patients (14.5\%) in group A developed a complete AV block necessitating the implantation of a permanent pace maker while only two patients $(3.2 \%)$ in group $\mathrm{B}$ needed a permanent pace maker implantation. Binary logistic regression showed that the operative technique was the only independent predictor of the need of permanent pacemaker implantation. The odds of needing pace maker implantation were 5 times higher among patients in group A as compared to patients in group B. Patient's age has been linked to the incidence of PPMI with an OR of 1.13, 95\%confidence interval (CI) 0.99 to 1.27 however it failed to reach statistical significance (table 3 ).

Table 3. Logistic regression showing predictors of the need of permanent pace maker after surgery.

\begin{tabular}{llll}
\hline & OR & $\mathbf{9 5 \% C I}$ & P value \\
\hline Method (Classic vs. New) & 5.4 & $1.10-27.0$ & 0.04 \\
Age & 1.13 & $0.99-1.27$ & 0.055 \\
\hline
\end{tabular}

\section{Discussion}

In the last years, a significant increase in the number of patients referred for surgical aortic valve replacement has been noticed due to a larger aging population and an improved diagnosis of valvular disease [1].

Conduction disturbances requiring pace maker implantation has been encountered after surgical aortic valve replacement. The incidence of complete AV block requiring PPMI reached $11.8 \%$ in some studies [1-3].

Although perioperative risk predictors for PPI following AVR are not well defined, many factors has been incriminated such as previous aortic regurgiatation, myocardial infarction, pulmonary hypertention, preoperative use of calcium channel blockers, postoperative electrolytes imbalance, prolonged aortic clamp and bypass time, large size of implanted valve prosthesis and endocarditis [1-4].

However, many papers attribute the rhythm problem after AVR to a significant trauma to the conduction system. In fact, extensive debridement of valve leaflets, placement of sutures, removal of calcium from the membranous septum and right trigone beneath the non coronary cusp-right coronary cusp commissure may precipitate conduction abnormalities leading to complete AV block post operatively $[1,4]$.

In order to minimize the trauma to the $\mathrm{AV}$ conduction system we modified the surgical technique which is classically used in such procedures. In fact, when the classic technique is performed, the sutures used are pledgeted 2.0 ethibond with the pledgets applied below the annulus while when performing the modified technique, the same 2.0 pledgeted ethibond sutures are put below the annulus except for 3-4 sutures at the commissure between the right and non coronary sinuses. They are applied above the annulus in order to avoid disturbances of the conduction system.

The impact of the new technique on the incidence of PPMI after valve replacement was evaluated in this study. After analyzing all the variables that might affect the incidence of PPMI, we found that the adoption of the modified technique was the unique independent factor that was linked to the lower incidence of AV block and subsequently the need for PPMI observed in the group of patients who underwent the new technique $(p<0.005)$. This result encourages us to adopt this technique in all the patients candidates for surgical aortic valve replacement.

However, many previous studies found that many factors were responsible of increasing the incidence of PPMI after surgical AVR; Erdogan et al found that annular calcifications, female gender, previous conduction abnormalities, hypertension and prolonged total perfusion time were risk factors for PPMI after AVR [4]. Limongelli et al suggest that previous aortic regurgitation, myocardial infarction pulmonary hypertension and postoperative electrolyte imbalance should be considered in order to identify patients at risk for advanced AV block [5]. Bagur and colleagues found that preexisting bundle branch block predicted the need for PPMI [6]. In contrast Nardi et al found that the need for PPMI after AVR was related more to preoperative advanced aortic valve disease rather than preexisting conduction system abnormalities [7]. All these variables were analyzed in our study and did not seem responsible of increasing the need for PPMI following aortic valve replacement. However the patient's age seemed to have some impact on the incidence of PPMI post AVR with an odds ratio of $1.13(\mathrm{p}=0.056)$.

Although this study showed that the significant decrease of PPM requirement post AVR was mainly due to the adoption of the new technique, it analyzes the data of only 125 patients that were operated in one center. This small cohort of patients may be considered as a limitation in our study. Therefore additional multicenters research and studies may be necessary to support the findings described in this paper.

To note that we consider as a strength of our work the fact that the study was conducted in one center and all the procedures were performed by one surgeon which limits the bias that might be linked to surgical skills.

On the other hand, The relative low incidence of PPMI post AVR observed following the new surgical technique of aortic valve replacement must be taken into consideration in new techniques performed in aortic valve procedures. The transcatheter aortic valve replacement (TAVR) technique have been adopted increasingly all over the world in the recent last years in high and intermediate risk candidates and is currently evaluated even in low risk patients. Atrioventricular conduction anomalies necessitating the implantation of permanent pace maker is one of the major adverse events of this technique with an incidence of PPMI 
reaching $36 \%$ in some series $[3,6]$. Since this incidence is relatively high in TAVR and since this incidence has been decreased to $3.2 \%$ in the patients undergoing the modified technique described in this paper, the selection of patients for TAVR must take into consideration this incidence and adopt it as a benchmark whenever patients- especially intermediate and low risk candidates- are offered this less invasive option.

\section{Conclusion}

Conduction disturbances requiring permanent pacemaker implantation was observed following SAVR and TAVR. In order to decrease the need of PPMI in patients undergoing SAVR we developed a modified surgical technique that we performed on 63 patients. We compared the incidence of PPMI following this new technique to the PPMI need in a group of 62 patients who underwent the classic SAVR. The incidence was significantly lower in the group of patients who underwent the modified technique (3.2\% versus $14.5 \%)$.

This low incidence encourages us to recommend this technique in all patients undergoing biological SAVR especially that this technique is simple to apply highly reproducible and reliable. However, further multicenter and larger studies will help confirm our findings.

\section{References}

[1] Klapkowski A, Pawlaczyk R, Kempa M, Jagielak D et al. Complete atrioventricular block after isolated aortic valve replacement. Kardiol pol 2016; 74: 985-993.

[2] Matthews IG, Fazal IA, Bates M, Turley A. In patients undergoing aortic valve replacement, what factors predict the requirement for permanent pacemaker implantation?

[3] Hwang YM, Kim J, Lee HL, Kim M et al. Conduction disturbances after isolated surgical aortic valve replacement in degenerative aortic stenosis. The journal of Thoracic and cardiovascular surgery2017; 154: 1556-1566.

[4] Erdogan HB, Kayalar N, Ardal H, Omeruglu SN et al. Risk factors for requirement of permanent pacemaker implantation after aortic valve replacement. J Card Surg 2006; 21:211-215.

[5] Limomgelli G, Ducceschi V, D ‘Andrea A, Renzulli A, Sarrubi
B, De Feo M, Cerasuolo F, Calabro R, Cotrufo M: Risk factors for pacemaker implantation following aortic valve replacement: a single centre experience. Heart, 2003; 89: 901904.

[6] Bagur R, Manazzoni JM, Dumont E, Doyle D et al: Permanent pacemaker implantation following isolated aortic valve replacement in a large cohort of elderly patients with severe aortic stenosis. Heart 2011; 97: 1687-1694.

[7] Nardi P, Pellegrino A, Scafuri A, Bellos K et al. Permanent pacemaker implantation after isolated aortic valve replacement: incidence, risk factors and surgical technical aspects. Journal of cardiovascular medicine 2010; 11: 14-19.

[8] Dawkins S, Hobson A, Kalra P, Tang A et al. Permanent pacemaker implantation after isolated aortic valve replacement: incidence, indications and predictors. Ann thorac Surg 2008; 85: 108-112.

[9] Glauber M, Lio A, Miceli A. Sutureless technology for aortic valve replacement: Looking beyond crossclamp time. J Thorac Cardiovasc Surg. 2016; 151: 1637-1638.

[10] Yanagawa B, Cruz J, Boisvert L, Bonneau D. A simple modification to lower incidence of heart block with sutureless valve implantation. Eur Heart J. 2013; 34: 1894-1905.

[11] Kostopoulo A, Karyofillis P, Livanis E et al. Permanent pacing after transcatheter aortic valve implantation of a corevalve prosthesis as determined by electrocardiographic and electrophysiological predictors: a single-centre experience. Europace, 2016; 18: 131-137. doi: 10.1093/europace/euc137.

[12] Van der Boon R, Houthuizem P, Urena M et al. Trends I $n$ the occurrence of new conduction abnormalities after transcatheter aortic valve implantation. Catheter Cardiovasc Intervent. 2015; 85: 144-152. doi: 10.1002/cod.25765.

[13] Onalan O, Crystal A, Lashevsky I, Khalameizer V et al. Determinants of pacemaker dependency after coronary and/or mitral or aortic valve surgery with long term follow-up. Am J Cardiol 2008; 101: 203-208.

[14] Merin O, Dearini J, Hyberger L Schaff $\mathrm{H}$ et al. Indications, effectiveness and long -term pacing dependency after cardiac surgery. Am J Cardiol 1997; 80: 1309-1313.

[15] Toggweiler S, Kobza R. Pacemaker implantation after transcatheter aortic valve: Why is this still happenning? J Thorac Dis 2018; 10 (Suppl 30): 3614-3619. doi: 10.21037/jtd.2018.06.103. 